\title{
IDENTIFICAÇÃO DA SÍNDROME DE BURNOUT EM MOTORISTAS DO TRANSPORTE DA SAÚDE
}

\author{
Caroline Estéfani Zanin \\ Universidade do Oeste de Santa Catarina \\ Amanda Saraiva Angonese \\ Universidade do Oeste de Santa Catarina
}

\begin{abstract}
Resumo
O estudo objetivou verificar a ocorrência da Síndrome de Burnout nos Motoristas do Transporte da Saúde, analisando-se as consequências que esta pode trazer para a vida do profissional. A pesquisa é de abordagem quanti-qualitativa e de caráter descritivo. Aplicou-se o formulário do Maslach Burnout Inventory (MBI-HSS) para identificação da síndrome e uma entrevista semiestruturada. Participaram da investigação 14 Motoristas da Saúde dos municípios que compõem a Agência de Desenvolvimento Regional de município do interior do estado de Santa Catarina. Para a análise dos dados relativos ao instrumento MBI-HSS, foram seguidas as normas apresentadas pelo instrumento e, para a análise dos dados da entrevista, foi utilizada a técnica de análise de conteúdo de Bardin (2009). Os resultados apontaram a manifestação da Síndrome de Burnout nessa categoria de trabalhadores ( $93 \%$ dos entrevistados). Conclui-se que a manifestação da síndrome neste público ocorre pela profissão ocasionar intenso estresse e exposição a fatores nocivos.
\end{abstract}

Palavras-chave: burnout; trabalho; doenças profissionais; esgotamento emocional; psicologia do trabalho.

\section{IDENTIFICATION OF BURNOUT SYNDROME IN HEALTH TRANSPORT DRIVERS}

\begin{abstract}
The study aimed to verify the occurrence of Burnout Syndrome in Health Transport Drivers, analyzing its consequences to the life of the professional. The research is quantitative-qualitative and descriptive in nature. The Maslach Burnout Inventory (MBI-HSS) form was applied to identify the syndrome and a semi-structured interview. The participants of the study were 14 Health Drivers from the municipalities that make up the Regional Development Agency of a municipality in the interior of the state of Santa Catarina. For the analysis of the MBI-HSS data, the standards presented in the instrument were followed and for data analysis of the interview, the technique of content analysis of Bardin (2009) was used. The results pointed to the manifestation of Burnout Syndrome in this category of workers ( $93 \%$ of respondents). We concluded that the manifestation of the syndrome in this public occurs because the profession causes intense stress and exposure to harmful factors.
\end{abstract}

Keywords: burnout; job; professional diseases; emotional exhaustion; psychology of work. 


\title{
IDENTIFICACIÓN DEL SÍNDROME DE BURNOUT EN MOTORISTAS DEL TRANSPORTE DE LA SALUD
}

\begin{abstract}
Resumen
El estudio tuvo como objetivo verificar la ocurrencia del síndrome de Burnout en los conductores del transporte sanitario, analizando sus consecuencias para vida del profesional. La investigación es de naturaleza cuantitativa-cualitativa y descriptiva. Se aplicó el formulario Maslach Burnout Inventory (MBI-HSS) para identificar el síndrome y una entrevista semiestructurada. En el estudio participaron 14 Promotores Sanitarios de los municipios que integran la Agencia de Desarrollo Regional de un municipio del interior del estado de Santa Catarina. Para el análisis de los datos de MBI-HSS, se siguieron los estándares presentados en el instrumento y para el análisis de datos de la entrevista, se utilizó la técnica de análisis de contenido de Bardin (2009). Los resultados apuntan la manifestación del Síndrome de Burnout en esta categoría de trabajadores ( $93 \%$ de los encuestados). Concluimos que la manifestación del síndrome en este público ocurre porque la profesión causa estrés intenso y exposición a factores dañinos.
\end{abstract}

Palabras clave: quemadura; trabajo; enfermedades profesionales; agotamiento emocional; psicología del trabajo.

\section{INTRODUÇÃO}

"O ser humano é capaz de adaptar-se ao meio ambiente desfavorável, mas esta adaptação não acontece impunemente." Lennart Levy

O trabalho é operador fundamental na própria construção do sujeito. O sujeito pensa sua relação com o trabalho, produz interpretações acerca de sua situação e as socializa através de atos intersubjetivos. Reage, se organiza e age sobre o próprio processo de trabalho, ocorrendo assim uma contribuição à construção e evolução das relações sociais no trabalho. O trabalho é um mediador privilegiado entre o inconsciente e o campo social, entre a ordem singular e a ordem coletiva e para a construção da saúde mental (Dejours \& Abdoucheli, 1994).

Além de possibilitar transformação, satisfação, segurança, independência pessoal e financeira, autonomia, crescimento pessoal e profissional para o indivíduo, e ser fonte de experiências de relações humanas satisfatórias, o trabalho e sua dinâmica também podem ocasionar insatisfação, estresse, irritabilidade, insônia e doenças físicas e psicológicas. O ambiente de trabalho exerce sobre o indivíduo ações específicas, cujo impacto se dá no aparelho psíquico (Mendes, 1995). No caso dos Motoristas do Transporte da Saúde, vários são os fatores do ambiente de trabalho que podem influir direta e negativamente em suas vidas, tais como características do veículo, trânsito, condições das vias, fatores climáticos, duração da jornada de trabalho, poluição sonora e visual, poluentes químicos, e o contato com pacientes em estado crítico e/ou com risco de vida (Fonte, 2010; Pinto \& Souza, 2015).

De acordo com Battiston, Cruz e Hoffmann (2006), a atividade de dirigir é desgastante, causa fadiga e sua eficácia está relacionada, principalmente, a fatores ambientais do local de trabalho e à forma como os motoristas 
desenvolvem estratégias de enfrentamento para lidar com esses fatores. A condição de trabalho interfere no estado psicofisiológico do motorista, traduzindo-se em irritabilidade (que pode levar a um comportamento agressivo na direção), insônia (podendo resultar em sonolência nas horas de trabalho, diminuindo os reflexos), distúrbios cognitivos como os que afetam a atenção e o raciocínio (fator essencial para a direção segura) e alterações emocionais, como mudança de humor e tristeza (Battiston et al., 2006).

A ação de dirigir envolve uma complexa interação das funções psicológicas, físicas e cognitivas do motorista, pois conduzir um veículo envolve prática, conhecimento, memória, atenção, tomada de decisões em um ambiente repleto de informações, como tráfego de pedestres, de outros veículos, e diversidade de sons e imagens (Rozestraten, 1998). Ainda, especificamente no caso dos Motoristas da Saúde, a atenção e assistência aos pacientes que estão sendo conduzidos (Estado de Santa Catarina, 2012).

Ao Motorista da Ambulância são delegadas tarefas como conduzir imediatamente ao local do chamado, guiando o veículo com segurança; realizar manobras técnicas que garantam a sobrevivência do paciente até a chegada da equipe e/ou ao hospital; auxiliar, quando solicitado, a equipe médica e de enfermagem no atendimento ao paciente no local da ocorrência; transportar paciente em estado crítico, em maca, prancha ou similar, do local da ocorrência para o veículo e deste para o hospital (Estado de Santa Catarina, 2012). Cabe, ainda, ao Motorista da Saúde saber realizar atividades com veículos de diferentes portes, deslocando-se com segurança e no intervalo de tempo desejável ao atendimento de cada caso. Também se envolve na assistência à clientela (pacientes-familiares), reduzindo ao máximo possível os riscos para os transportados (Estado de Santa Catarina, 2012).

Embora os estudos referenciando à saúde mental dos Motoristas do Transporte da Saúde sejam escassos, os existentes nos apontam que estes apresentam um adoecer diferenciado da população em geral, visto que estão expostos a uma sucessão de fatores nocivos (físicos, químicos, biológicos), os quais podem desencadear doenças relacionadas ao trabalho, como a Síndrome de Burnout (Battiston et al. 2006; Fonte, 2010; Estado de Santa Catarina, 2012).

Conforme F. Lima et al. (2007), o termo burnout deriva do verbo inglês to burn out, que pode ser traduzido como "queima após desgaste". Caracterizado como um processo de esgotamento, o termo passou a ser usado como metáfora para explicar o sofrimento do profissional em seu ambiente de trabalho, associado a uma perda de motivação e alto grau de insatisfação, decorrentes do prolongado estresse profissional.

Burnout se refere a um conjunto de sinais e sintomas agrupados em três dimensões distintas: Exaustão Emocional, que se refere ao sentimento de sobrecarga e desgaste emocional; Despersonalização, que diz respeito aos 
sentimentos negativos em relação ao próximo e às atitudes de ironia e cinismo com o outro; e a baixa Realização Profissional, que está relacionada com os sentimentos de inadequação pessoal e profissional ao trabalho. Como é um processo que se desenvolve sequencialmente, as três dimensões devem ser consideradas para que se possa caracterizar a síndrome (Maslach \& Jackson, 1986).

No cenário mundial, em diversos países, sobretudo Estados Unidos da América/EUA, Holanda e Inglaterra, verifica-se o aumento de casos de estresse relacionado ao trabalho e estima-se que a Síndrome de Burnout seja responsável por 50-60\% dos casos de absenteísmo (Cox, Griffiths, \& Rial-González, 2000; Grau-Alberola, Gil-Monte, Juesas, \& Ferraz, 2009; Schaufeli \& Enzmann, 1998). Avalia-se que pelo menos $10 \%$ da população trabalhadora dos EUA, Reino Unido, Alemanha e Polônia são acometidos por depressão, ansiedade, estresse e Síndrome de Burnout (International Labour Organization, 2000, citado por A. S. Lima, 2016, p.14). No Canadá, um estudo evidenciou que enfermeiros possuíam uma das taxas mais altas de licenças médicas entre todos os trabalhadores, o que se devia, principalmente, ao burnout, ao estresse induzido pelo trabalho e às lesões musculoesqueléticas. Na Alemanha, um levantamento estimou que 4,2\% dos trabalhadores de sua população eram acometidos pela síndrome (Trigo, Teng, \& Hallak, 2007). Já no Brasil, segundo pesquisas realizadas pela International Stress Management Association no Brasil (2013), 70\% dos brasileiros sofrem de estresse, sendo que, deste total, 30\% sofrem de burnout.

A Síndrome de Burnout pode manifestar-se clinicamente por meio de quatro classes sintomatológicas distintas. As manifestações físicas ocorrem quando o trabalhador apresenta fadiga constante, distúrbio do sono, falta de apetite e dores musculares generalizadas ou inespecíficas. As manifestações psíquicas são caracterizadas pela falta de atenção, alterações de memória, ansiedade e frustração. As alterações comportamentais são identificadas através de negligência no trabalho, irritabilidade ocasional e instantânea, diminuição da capacidade de concentração, aumento de incidentes conflitivos com os colegas de trabalho, necessidade de longas pausas para o descanso e o cumprimento irregular da carga horária de trabalho. O comportamento defensivo ocorre quando o trabalhador apresenta a tendência ao isolamento, sentimento de onipotência e empobrecimento da qualidade do trabalho prestado (Carlotto, 2001; F. Lima et al., 2007).

Em suma, a Síndrome de Burnout seria resultante de um conflito entre trabalhador e trabalho, culminando com um desgaste emocional e físico caracterizado pelo aumento de sentimentos de sobrecarga e exaustão emocional, tendência à avaliação negativa do próximo e insatisfação profissional. Além disso, a síndrome seria mais comum em profissões de natureza assistencial, devido ao contato direto com pessoas (Maslach \& Jackson, 1981). 
O presente estudo se justificou pela importância de compreender o modo de trabalhar dos Motoristas do Transporte da Saúde, na medida que se observa sua ação profissional na sociedade com a atribuição de auxiliar a salvar e manter vidas, defrontando-se com o barulho da sirene, riscos de acidentes, más condições das vias, rapidez de velocidade do veículo, fatores climáticos, atenção constante aos sinais de trânsito e a outros veículos, e aos pacientes em estado crítico. Assim, um estudo sobre o desgaste crônico, físico e psicológico sofrido pelos Motoristas do Transporte da Saúde desperta o interesse por se tratar de ambientes e ocasiões propícias ao desenvolvimento da Síndrome de Burnout.

Os benefícios propiciados a partir da presente pesquisa serão informações sobre a dinâmica de trabalho do Motorista do Transporte da Saúde e conhecimento amplificado sobre o estado de saúde dessa categoria de profissionais. Espera-se atrair a atenção dos gestores de saúde, para que possam proporcionar condições de trabalho que sejam adequadas a essa categoria profissional, atribuindo-Ihes o devido reconhecimento.

Frente à importância do gerenciamento e cuidado da saúde desses profissionais, o objetivo da pesquisa foi verificar a ocorrência da Síndrome de Burnout em Motoristas do Transporte da Saúde nos sete municípios de abrangência da Agência de Desenvolvimento Regional (ADR) de um município do interior do estado de Santa Catarina, identificando variáveis responsáveis pelo desencadeamento da Síndrome de Burnout nessa categoria de trabalhadores, bem como analisar quais as consequências que essa síndrome pode trazer para a qualidade de vida do profissional no trabalho, segundo suas percepções, e em suas relações intra e interpessoais.

\section{MÉTODO}

Caracterização da pesquisa

A pesquisa é de abordagem quanti-qualitativa, de caráter descritivo. Sendo os participantes escolhidos por conveniência.

\section{Participantes}

Foram convidados a participar da investigação 15 Motoristas do Transporte da Saúde, funcionários públicos, dos sete municípios que compõem a Agência de Desenvolvimento Regional (ADR) de um município do interior do estado de Santa Catarina. O critério de inclusão foi o tempo de serviço, sendo que este não poderia ser inferior a dois anos.

Os entrevistados foram abordados em seus locais de trabalho. A aplicação do instrumento MBI-HSS e entrevista ocorreram de forma verbal, com anotações das respostas mencionadas pelos mesmos, de maneira individual.

Assim, a amostra constituiu-se de 14 Motoristas do Transporte da Saúde, pois um profissional recusou-se a participar do estudo. 
A escolha do público-alvo da pesquisa se justifica porque os profissionais mais suscetíveis ao desenvolvimento da síndrome são os de natureza assistencial (Benevides-Pereira, 2002; Formighieri, 2003; Volpato et al. 2003), que trabalham diretamente em contato com outras pessoas, muitas vezes cuidando delas, como no caso dos profissionais de saúde (médicos, enfermeiros) ou tendo, simplesmente, que lidar com elas, como no caso dos profissionais de educação (professores) (F. Lima et al., 2007). Ademais, a investigação estende-se a todos os grupos ocupacionais, incluindo estudantes (Leiter \& Schaufeli, 1996; Maslach, Schaufeli \& Leiter, 2001; Schaufeli \& Janczur, 1994).

\section{Instrumento}

Para a coleta dos dados, primeiramente, foi aplicado o formulário do Maslach Burnout Inventory (MBI-HSS), composto por 22 perguntas que avaliam as três dimensões da Síndrome de Burnout (Exaustão Emocional, Despersonalização e Realização Profissional) (Maslach \& Jackson, 1986). O MBIHSS foi o primeiro instrumento a ser criado para avaliar a incidência da Síndrome de Burnout, elaborado em um estudo com profissionais de serviços sociais e de saúde, em que se constatou a identificação da síndrome nesses profissionais, juntamente com atitudes negativas e de distanciamento pessoal (Farber, 1991; Maslach \& Jackson, 1981). Inicialmente, o inventário possuía 47 itens, que foram administrados em uma amostra de 605 sujeitos de ocupações tais como enfermagem, medicina e assistência social. Por meio de avaliações e adaptações criteriosas, o MBI-HSS passou a quatro dimensões, distribuídas em 25 itens, sendo respondido por uma escala Likert de frequência (de 0 "nunca" à 6 "todos os dias"). Após novas pesquisas, o número de itens passou a 22 , tendo sido retiradas as respostas de intensidade, e o instrumento passou a conter três dimensões, que apresentaram significância empírica (Farber, 1991; F. Lima et al. 2007; Maslach \& Jackson, 1981).

O MBI-HSS, no decorrer dos anos, passou por um longo processo de validação em vários países, como Holanda, Espanha, Portugal (Maroco, Tecedeiro, Martins, \& Meireles, 2008; Schaufeli, Martinez, Pinto, Salanova, \& Bakker, 2002), China (Hu \& Schaufeli, 2009), Canadá e Estados Unidos da América (Trigo et al. 2007). Em suma, o MBI-HSS é o inventário mais utilizado mundialmente para avaliação de burnout (Benevides-Pereira, 2012). No Brasil, a Síndrome de Burnout está contemplada entre as Doenças do Trabalho, na descrição dos Agentes Patogênicos causadores de Doenças Profissionais, da Presidência da República (1999). E, apesar de possuir diversas traduções e adaptações para o Brasil (Benevides-Pereira, 2001; Lautert, 1995; Roazzi, Carvalho \& Guimarães, 2000; Tamayo 1997), a partir da Resolução 09/2018 do Conselho Federal de Psicologia (2018), determina-se que o inventário pode ser utilizado apenas com o intuito de pesquisa. Para tanto, a versão utilizada na pesquisa foi Maslach Burnout Inventory-Human Services Survey (MBI-HSS) e o 
manual para correção desse instrumento baseou-se no trabalho científico $M B I-$ Maslach Burnout Inventory e suas adaptações para o Brasil de Benevides-Pereira (2001).

Em seguida, encerrando-se a coleta de dados, foram realizadas entrevistas semiestruturadas, com perguntas abertas, elaboradas pelos autores da pesquisa, para o aprofundamento das informações obtidas no instrumento. Todas as entrevistas ocorreram de maneira individual, sendo aplicadas no local de trabalho dos participantes que aceitaram participar da pesquisa.

\section{Plano de Análise de Dados}

Para a análise dos dados relativos ao instrumento MBI-HSS, foi realizada a somatória de cada dimensão de acordo com as normas de correção apresentadas pelo próprio instrumento. Em seguida, os valores obtidos foram comparados com os valores de referência, sendo relativos às dimensões Exaustão Emocional, Despersonalização e Realização Profissional. A classificação das três dimensões ocorre em três níveis: baixo, médio e alto. O manual do MBI-HSS traz como princípio para o diagnóstico de burnout a obtenção de nível alto para Exaustão Emocional e Despersonalização e nível baixo para Realização Profissional. Portanto, o enquadramento do profissional nesses três critérios dimensionais indica a manifestação de burnout (Benevides-Pereira, 2001; Menegaz, 2004). Por fim, foi realizada a discussão dos resultados com base na análise e interpretação das informações obtidas.

Já para a análise dos dados da entrevista, foi utilizado o método de análise de conteúdo, que se configura como um conjunto de técnicas de análise das comunicações, fazendo uso de procedimentos sistemáticos e objetivos de descrição do conteúdo das mensagens (Bardin, 2009). Desse modo, a análise de conteúdo pode ser organizada levando-se em consideração três eixos: préanálise, exploração do material e tratamento dos resultados (Bardin, 2009).

Na pré-análise, realizou-se a organização do material a ser examinado, por meio de uma leitura geral das entrevistas, que aos poucos se tornou mais consistente, objetiva e precisa, e configurou-se no segundo eixo, a exploração do material. Nesta etapa, realizou-se uma releitura mais atenta, destacando trechos que despertaram interesse pela relação que as falas dos entrevistados apresentavam com os objetivos da pesquisa. Finalmente, foi realizada a classificação e a agregação dos dados, escolhendo as categorias e subcategorias teóricas ou empíricas, responsáveis pela especificação do tema (Bardin, 2009). Após esse processo, foram realizadas inferências e interpretações, atribuindo significados aos resultados finais por meio da análise qualitativa dos dados e da reflexão com embasamento nos materiais empíricos.

Dessa forma, foram definidas as categorias e subcategorias. As categorias definidas foram: Caracterização dos Entrevistados e Dinâmica do Trabalho. As subcategorias foram assim definidas: sexo, estado civil, escolaridade, e tempo de 
serviço, para a categoria Caracterização dos Entrevistados; e fatores preditores à síndrome, pontos negativos, consequências, adoecimento físico e psicológico, pontos positivos da função, e família x trabalho, para a categoria Dinâmica do Trabalho.

Assim, os resultados serão apresentados da seguinte maneira:

- Caracterização dos Entrevistados.

- Manifestação da Síndrome de Burnout em Motoristas do Transporte da Saúde.

- Dinâmica do Trabalho do Motorista do Transporte da Saúde.

As variáveis encontradas serão apresentadas na forma de frequência relativa e frequência absoluta.

\section{Considerações Éticas}

A importância dessa pesquisa se explicita também no cuidado e no rigor com que se propõe a tratar dos sujeitos envolvidos. Sendo assim, os mesmos foram esclarecidos sobre a temática de que trata a pesquisa, cabendo a eles a decisão de participar ou não. Todos tiveram acesso ao projeto, foram consultados previamente, e tiveram acesso ao Termo de Consentimento Livre e Esclarecido (TCLE), em duas vias, sendo o mesmo assinado pelos participantes.

O projeto deste estudo foi submetido e aprovado pelo Comitê de Ética em Pesquisa Humana da Universidade do Oeste de Santa Catarina - UNOESC, em 04 de outubro de 2016, conforme número do parecer: 1.760.458.

\section{RESULTADOS E DISCUSSÃO}

Caracterização dos entrevistados

Analisando os dados sociodemográficos, observou-se que houve a unanimidade do sexo masculino, sendo que destes, 08 (57\%) são casados e 06 (43\%) são solteiros; 04 (29\%) têm carga horária semanal de trabalho entre 40 e 70 horas e 10 (71\%) têm carga horária equivalente a 80 horas semanais. A Consolidação das Leis do Trabalho (CLT) prevê a jornada de trabalho de, no máximo, 08 horas diárias, totalizando 44 horas semanais. Quando o funcionário ultrapassa a quantidade de horas especificadas em contrato, há o pagamento pelas horas excedentes, e o limite de horas trabalhadas, não poderá ultrapassar 02 horas diárias (Direitos Trabalhistas, 2017). Nesse sentido, observa-se que os profissionais entrevistados possuem excessiva carga horária de trabalho, pois, além da jornada de trabalho regular, realizam horas extras remuneradas além do limite previsto na CLT e também realizam plantões semanais com jornada de 24 horas. Esse dado é verificado em outros estudos referentes à burnout, em que constata-se carga horária de trabalho dos entrevistados ultrapassando as 40 horas semanais, bem como sentimentos de sobrecarga e, por consequência, a predisposição para a manifestação de burnout no público estudado (Dalri, Silva, 
Mendes, \& Robazzi, 2014; Jodas \& Haddad' 2009; Murofuse, Abranches, \& Napoleão, 2005; Pereira \& Mello, 2017). Alguns estudos na área da saúde e da Psicologia Organizacional apontam o excesso de horas extras realizadas pelo trabalhador como uma das principais fontes causadoras de estresse e, consequentemente, do desencadeamento da Síndrome de Burnout (Álvarez, 2011; Ministério da Saúde, 2010; Campos, 2005; Jodas \& Haddad, 2009; Menegaz, 2004; Tamayo \& Tróccoli, 2002).

Quanto à escolaridade, $02(14 \%)$ os Motoristas relataram ter o Ensino Fundamental Incompleto, 07 (50\%) o Ensino Fundamental Completo, 04 (29\%) o Ensino Médio Completo, e 01 (7\%) o Ensino Superior. Com relação ao tempo de serviço dos entrevistados, o mesmo varia de 02 a 22 anos, sendo a maior incidência entre 02 e 12 anos. Essa informação nos revela também que há significativa rotatividade de pessoal nesse setor. Apenas 03 (21\%) dos 14 entrevistados estão há mais de 12 anos na função. O motivo dessa rotatividade se explicita na fala dos entrevistados, onde os mesmos mencionam que muitos colegas, que estavam há anos atuando como Motoristas do Transporte da Saúde, estão afastados do trabalho devido à ocorrência de doenças físicas e psicológicas ocasionadas pelo mesmo, tais como Síndrome do Pânico e úlceras. Pode-se considerar que, conforme verificado em outros estudos, trabalhadores com menor tempo de atuação apresentam maior predisposição a sofrer com a Síndrome de Burnout, devido a expectativas falhas quanto às suas atribuições e, também, à falta de socialização no trabalho com seus pares, abandonando a carreira com pouco tempo de atuação, enquanto aqueles trabalhadores com maior tempo de profissão podem desenvolver mecanismos de enfrentamento que possibilitam uma maior tolerância às dificuldades do serviço (Freudenberger, 1974; Maslach \& Jackson, 1981; Maslach et al., 2001).

Assim, com base nos dados obtidos e na revisão da literatura, pode-se considerar que a manifestação da Síndrome de Burnout em Motoristas da Saúde ocorre devido à profissão ocasionar intenso estresse (Battiston et al., 2006; Fonte, 2010; Pinto \& Souza, 2015). O esgotamento no trabalho, bem como o estresse, estão relacionados à Síndrome do Pânico, depressão e também doenças gastrointestinais (Jornal R7, 2015; Peixoto, 2016). De acordo com Chagas (2017), as doenças mentais respondem pela terceira maior causa de afastamento do trabalho no Brasil. Também é verificado que a Síndrome de Burnout provoca elevados custos financeiros para as organizações, e essas implicações financeiras são avaliadas principalmente diante de absenteísmo, rotatividade e aposentadoria precoce (Trigo et al., 2007).

\section{Manifestação da Síndrome de Burnout em Motoristas do Transporte da Saúde}

Tratando-se dos valores encontrados em cada dimensão da síndrome, constatou-se que 13 (93\%) dos 14 profissionais entrevistados possuem alto nível (escores entre 26 e 54) de Exaustão Emocional; estes 13 (93\%) também 
possuem nível alto (escores entre 09 e 30) de Despersonalização e um baixo nível (escores entre 0 e 33) de Realização Profissional, características essas que estabelecem diagnóstico para manifestação de burnout de acordo com o MBIHSS. E apenas 01 motorista (7\%), atuando na função há 02 anos, obteve médio risco para o desencadeamento da síndrome. Nenhum profissional entrevistado manifestou baixo risco para a manifestação da mesma.

Considerando o diagnóstico da Síndrome de Burnout (13 profissionais, 93\%) no público estudado, bem como a indicação de médio risco para o desencadeamento da mesma (01 profissional, 7\%), a literatura destaca a Psicologia e suas ferramentas (psicoterapia individual e grupal, bem como intervenções psicológicas no ambiente organizacional) como uma excelente estratégia na prevenção e tratamento da Síndrome de Burnout (Frazão, 2014; Varella, 2014). O profissional da área se direcionará para a compreensão do modo como os fatores biológicos, comportamentais e sociais influenciam no bem-estar do trabalhador, centrando-se na promoção da saúde e prevenção de doença, trabalhando com os fatores psicológicos que fortalecem a saúde e que reduzem o risco de adoecer, podendo ser disponibilizados através de serviços clínicos individuais e grupais (Teixeira, 2004).

Vale salientar que não houve diferença entre os resultados obtidos nos profissionais que estão há menos tempo na função em comparação com aqueles que estão há mais tempo, pois em ambas as categorias os resultados foram significativos termos quantitativos, colocando a saúde dessa categoria de trabalhadores em alerta. Para essa análise, utilizou-se o teste não-paramétrico, comparando-se as duas categorias e observando-se a significância de cada uma. Conforme Freudenberger (1974), a síndrome começa a se manifestar a partir do primeiro ano de trabalho e incide, principalmente, nos que ajudam, prestam assistência ou são responsáveis, de certa maneira, pelos outros.

\section{Dinâmica do trabalho do Motorista do Transporte da Saúde}

Quanto aos fatores preditores para o desenvolvimento de burnout, o fato de desempenhar atividades que exigem excessiva carga horária de trabalho, o tipo de paciente atendido, bem como a desvalorização do profissional e de sua função, o cansaço, a falta de apoio médico e psicológico e a ausência de incentivo, são fatores que se destacam nas falas dos entrevistados como principais causas para a manifestação da síndrome, conforme os exemplos que se seguem:

\footnotetext{
"Jornada de trabalho excessiva, não há acompanhamento médico nem psicológico. Falta apoio da Gestão. Estou esgotado". (Entrevistado 1)

"Incompreensão da Gestão e de outros profissionais da Saúde. Somos desvalorizados". (Entrevistado 6)

"Lidar com pacientes doentes, crianças com câncer, o trânsito estressante e não temos nenhum amparo". (Entrevistado 7)
} 
"Vida estressante. Tenho insônia, estou sempre tenso, nervoso". (Entrevistado 3)

A sobrecarga de função e carga horária de trabalho tem sido umas das variáveis mais apontadas em outros estudos como predisponentes a burnout (Benevides-Pereira, 2008; Ministério da Saúde, 2010; Campos, 2005; Jodas \& Haddad, 2009; Menegaz, 2004; Tamayo \& Tróccoli, 2002). A natureza da relação entre paciente e trabalhador, bem como o tipo de problema/doença do paciente podem afetar o profissional (Carlotto, 2001). Pacientes multiqueixosos, depressivos, em situações de risco e suicidas são tidos como especialmente difíceis. Também, o contato com o sofrimento e a morte, principalmente de crianças, tem sido referido como umas das principais causas de burnout em profissionais que atuam na área da saúde (Maslach, Schaufeli, \& Leiter, 2001).

A sintomatologia da Síndrome de Burnout não traz consequências nocivas apenas para o indivíduo acometido por ela. Tais consequências podem atingir o indivíduo de diversas maneiras, interferindo nos níveis pessoal, organizacional e social, seja pela diminuição da qualidade do trabalho, absenteísmo, baixa produtividade laboral, acidentes de trabalho, isolamento, divórcio, desânimo, depressão, estresse, e também doenças físicas e psicológicas (Benevides-Pereira, 2001).

Nesse eixo, dos 14 entrevistados, 08 (57\%) necessitaram de atendimento especializado durante o tempo de atuação como Motorista do Transporte da Saúde. Ou seja, 05 (35\%) dos entrevistados foram a Especialista do Aparelho Digestivo, devido ao desencadeamento de gastrites e úlceras, 01 (7\%) a Ortopedista, devido a problemas no joelho, e 02 (14\%) a profissional da Psiquiatria devido a distúrbios do sono, irritabilidade e sentimentos negativos.

Estudos apontam uma extensa lista de sintomas/consequências associados à burnout. Esses sintomas podem ser subdivididos em físicos, psíquicos, comportamentais e defensivos (Benevides-Pereira, 2008; Carlotto, 2002; Lyra, 2015). Foram os seguintes os mencionados pelos entrevistados: cansaço, distúrbios do sono, enxaquecas, perturbações gastrointestinais, dores musculares e osteomusculares, impaciência, irritabilidade, entre outros.

Com relação aos pontos positivos da profissão apontados pelos entrevistados, destacam-se o conhecimento Geográfico, Social e de Saúde adquiridos, bem como novas amizades. Ainda, os motoristas destacaram a importância de suas famílias, considerando as mesmas como fator fundamental em suas vidas. As falas mais significativas a este respeito foram:

"A família é que dá forças para encarar o dia a dia estressante de trabalho". (Entrevistado 1)

"Olha, muitas vezes eu descontei meu estresse em minha família, fui impaciente, mas eles sempre estiveram do meu lado. São muito importantes, é o que motiva você a levantar todo o dia e ir trabalhar". (Entrevistado 9) 
A família do trabalhador é imprescindível na prevenção e/ou minimização da manifestação de burnout. O apoio familiar e o carinho que a família oferece aliviam as tensões e conflitos a que os trabalhadores se submetem em decorrência do trabalho (Moreira, Magnago, \& Magajewski, 2009). A possibilidade de poder contar com o apoio familiar e social é considerada um moderador efetivo no processo de burnout (Benevides-Pereira, 2001).

\section{CONSIDERAÇÕES FINAIS}

O estudo em questão possibilitou a identificação da manifestação da Síndrome de Burnout em Motoristas do Transporte da Saúde, bem como os fatores preditores e as consequências dessa síndrome na vida do trabalhador. Diante dos resultados apresentados, percebe-se a necessidade de atenção no gerenciamento da situação de saúde desses profissionais, considerando que têm maior proximidade físico-psicológica com o doente/familiares e exposição a fatores nocivos, como o veículo e o trânsito. Neste estudo, a maioria dos profissionais apresentou-se em estado de burnout (93\%), representando um resultado significativo e preocupante. Observou-se que a dinâmica do trabalho desses profissionais gera uma sobrecarga de movimento e tensão ocupacional, sendo necessário monitorar periodicamente a saúde mental e física desses trabalhadores, a fim de desenvolver estratégias que possam reorganizar o processo de trabalho, diminuindo as fontes de estresse. É necessário que medidas preventivas e de promoção à saúde sejam implementadas, a fim de reduzir a incidência e minimizar os efeitos da Síndrome de Burnout.

Portanto, sugere-se a adoção de estratégias organizacionais, individuais e de grupo, que promovam o aumento da qualidade de vida do profissional. Ou seja, a Secretaria de Saúde à qual o motorista está vinculado deve acompanhar os conflitos existentes na dinâmica de trabalho do profissional, disponibilizando espaços para discussão e ajuda, de modo a diminuir o estresse do trabalho. Aos gestores, sugere-se atenção especial ao favorecimento de condições de trabalho adequadas, que fomentem a saúde e o bem-estar dos Motoristas do Transporte da Saúde, ajustando a sobrecarga de trabalho, reconhecendo o trabalho e valorizando o profissional. A atuação do Psicólogo na organização do trabalho é uma excelente estratégia na prevenção da síndrome, através de grupos terapêuticos onde os trabalhadores possam se reunir para troca de informações, experiências, frustrações, insatisfações, estresses do cotidiano e as mais diversas questões relacionadas ao trabalho, desenvolvendo assim o apoio mútuo. Sugerese, também, horários para a realização de psicoterapia individual para ajudar o profissional a lidar com o estresse, formulando estratégias de enfrentamento positivas.

Os resultados apesentados devem ser analisados considerando-se algumas limitações do estudo, como: 1) amostra reduzida; 2) corte transversal, que 
inviabiliza estabelecer relações de causa e efeito; 3) investigação de validade da entrevista e; 4) ausência de grupo de controle. Entretanto, essas limitações não se mostraram impeditivas para o desenvolvimento desta pesquisa, uma vez que conseguiu-se responder aos objetivos propostos inicialmente, assim, tais limitações também não minimizam os resultados identificados. Além disso, sugere-se a realização de novos estudos sobre a temática, a fim de ampliar as informações, reflexões e ações sobre a saúde dos Motoristas do Transporte da Saúde, uma vez que estudos referenciando a saúde mental desses profissionais são esporádicos e os existentes colocam a mesma em alerta.

\section{DECLARAÇÃO DE CONFLITO DE INTERESSES}

Não há conflito de interesses.

\section{REFERÊNCIAS}

Álvarez R. F. (2011). El síndrome de burnout: Síntomas, causas y medidas de atención en la empresa. Éxito empresarial, (160), 1-4.

Bardin L. (2009). Análise de conteúdo. Lisboa, Portugal: Edições 70, LDA.

Battiston M., Cruz R. M., \& Hoffmann M. H. (2006). Condições de trabalho e saúde de motoristas de transporte coletivo urbano. Estudos de Psicologia, 11(3), 333-343. doi:10.1590/S1413-294×2006000300011

Benevides-Pereira, A. M. T. (2001). MBI - Maslach Burnout Inventory e suas adaptações para o Brasil. Anais da $32^{a}$ Reunião Anual de Psicologia (p. 8485). Rio de Janeiro, RJ: Congresso da Sociedade Brasileira de Psicologia.

Benevides-Pereira, A. M. T. (2002). Burnout: O processo de adoecer pelo trabalho. In A. M. T Benevides-Pereira, Burnout: Quando o trabalho ameaça o bem-estar do trabalhador (p. 21-91). São Paulo, SP: Casa do Psicólogo.

Benevides-Pereira, A. M. T. (2008). Burnout: Quando o trabalho ameaça o bemestar do trabalhador. São Paulo, SP: Casa do Psicólogo.

Benevides-Pereira, A. M. T. (2012). Considerações sobre a síndrome de Burnout e seu impacto no ensino. Boletim de Psicologia, 62(137), 155-168.

Campos, R. G. (2005). Burnout: Uma revisão integrativa na enfermagem oncológica. (Dissertação de Mestrado). Universidade de São Paulo, Ribeirão Preto, São Paulo, Brasil.

Carlotto, M. S. (2001). Síndrome de Burnout: Um tipo de estresse ocupacional. Canoas, RS: Cadernos Universitários Ulbra.

Carlotto, M. S. (2002). A Síndrome de Burnout e o trabalho docente. Psicologia em Estudo, 7(1), 21-29. 
Chagas, P. C. (2017). Transtornos mentais são a terceira maior causa de afastamento do trabalho. Brasília, DF: Agência Brasil. Recuperado de http://agenciabrasil.ebc.com.br/geral/noticia/2017-04/transtornos-mentaissao-terceira-maior-causa-de-afastamento-do-trabalho

Conselho Federal de Psicologia. (2018). CFP - Resolução 009/2018. Define e regulamenta o uso, a elaboração e a comercialização de testes psicológicos. Recuperado de http://satepsi.cfp.org.br/docs/Resolu\%C3\%A7\%C3\%A3oCFP-n\%C2\%BA-09-2018-com-anexo.pdf

Cox, T., Griffiths, A., \& Rial-González, E. (2000). Work-related stress. European Agency for Safety and Health at work. Recuperado de https://osha.europa.eu/en/tools-andpublications/publications/reports/203

Dalri, R. C. M. B., Silva L. M. A, Mendes, A. M. O. C., \& Robazzi, M. L. C. C. (2014). Carga horária de trabalho dos enfermeiros e sua relação com as reações fisiológicas do estresse. Revista Latino-Americana de Enfermagem, 22(6), 959-65.

Dejours, C., \& Abdoucheli E. (1994). Itinerário Teórico em Psicopatologia do Trabalho. In: Dejours C, Abdoucheli E, Jayet C. Psicodinâmica do Trabalho. São Paulo, SP: Atlas.

Direitos Trabalhistas. (2017). Jornada de Trabalho. Disponível em: http://direitotrabalhista.info/direitos-do-trabalhador/jornada-de-trabalho.html

Estado de Santa Catarina. (2012). Regimento interno SAMU 192/SC. Florianópolis, SC: Secretaria de Estado da Saúde.

Farber, B. A. (1991). Crisis in education. Stress and Burnout in the american teachers. São Francisco, CA: Jossey-Bass Inc.

Fonte, E. (2010). Dispõe sobre a profissão de motorista e de condutor de veículos de emergência. Recuperado de http://www.camara.gov.br/proposicoesWeb/prop_mostrarintegra?codteor= 817110

Formighieri, V. J. (2003). Burnout em fisioterapeutas: Influência sobre a atividade de trabalho e bem-estar físico e psicológico. (Dissertação de mestrado). Universidade do Oeste de Santa Catarina, Florianópolis, Santa Catarina, Brasil.

Frazão, A. (2014). Síndrome de Burnout. Disponível em: http://www.tuasaude.com/sindrome-de-burnout/

Freudenberger, H. (1974). Staff burn-out. Journal of Social Issues, 30, 159-165. doi: $10.1111 /$ j.1540-4560.1974.tb00706

Grau-Alberola, E., Gil-Monte P. R., Juesas J. A. G., \& Ferraz, H. F. (2009). Efectos de los conflictos interpersonales sobre el desarrollo del síndrome de quemarse por el trabajo (burnout) y su influencia sobre la salud. Un estudio longitudinal en enfermería. Riesgos Psicosociales en el Trabajo, 11(32), 7279. 
Jornal R7. (2015). Esgotamento no trabalho pode causar Síndrome do Pânico e depressão. Recuperado de http://noticias.r7.com/saude/esgotamento-notrabalho-pode-causar-sindrome-do-panico-e-depressao-29082015

Hu, Q. \& Schaufeli, W. B. (2009). The factorial validity of the Maslach Burnout Inventory - student survey in China. Psychological Reports, 105(2), 394408.

International Stress Management Association no Brasil. (2013). Cansaço demais tem jeito. Isma-Br. Recuperado de http://ismabr.blogspot.com/2013/11/

Jodas, D. A. \& Haddad, M. C. L. (2009). Síndrome de Burnout em trabalhadores de enfermagem de um pronto socorro de hospital universitário. Acta Paulista de Enfermagem, 22(2), 192-197.

Lautert, L. (1995). O desgaste profissional do enfermeiro. (Tese de doutorado). Universidade Pontificia de Salamanca, Salamanca, Espanha.

Leiter, M. P. \& Schaufeli, W.B. (1996). Consistency of the Burnout constructo across ocupations. Anxiety Stress Coping, 9, 229-243.

Lima, A. S. (2016). Prevalência e fatores associados à Síndrome de Burnout nos profissionais da saúde da atenção primária de Juiz de Fora (Dissertação de Mestrado). Universidade Federal de Juiz de Fora, Juiz de Fora, Minas Gerais, Brasil.

Lima, F., Buunk, A.P, Araújo, M.B.J., Chaves, J.G.M., Muniz, D.L.O., \& Queiroz, L.B. (2007). Síndrome de Burnout em residentes da Universidade Federal de Uberlândia. Rio de Janeiro, RJ: Editora Med.

Lyra, G. J. H. (2015). Síndrome de Burnout: Esgotamento profissional, estresse, sintomas e o caminho para a liberdade docente. Revista Científica, 1,1-16.

Maroco, J., Tecedeiro, M., Martins, P., \& Meireles, A. (2008). Estrutura fatorial de segunda ordem da Escala de Burnout de Malasch para estudantes numa amostra portuguesa. Análise Psicológica, 10(2), 639-49.

Maslach, C. \& Jackson, S. E. (1986). The measurementof experienced burnout. Journal of Occupational Bahaviour, 2, 99-113.

Maslach, C. \& Schaufeli, W. B. \& Leiter, M. P. (2001). Job Burnout. Annual Review of Psychology, 52(1), 397-422.

Mendes, A. M. B. (1995). Aspectos psicodinâmicos da relação homem-trabalho: as contribuições de C. Dejours. Psicologia Ciência e Profissão, 15(1-3), 1-5. doi:10.1590/S1414-98931995000100009

Menegaz, F. D. L. (2004). Características da incidência de burnout em pediatras de uma organização hospitalar pública. (Dissertação de Mestrado). Universidade Federal de Santa Catarina, Florianópolis, Santa Catarina, Brasil.

Ministério da Saúde. Organização Pan-Americana da Saúde. (2010). Doenças relacionadas ao trabalho: Manual de procedimentos para os serviços de saúde. Brasília, DF: Ministério da Saúde. 
Moreira, D. S., Magnago, R. F., \& Magajewski, F. R. L. (2009). Prevalência da Síndrome de Burnout em trabalhadores de enfermagem de um hospital de grande porte da Região Sul do Brasil. Caderno Saúde Pública 25(7), 15591568. doi:10.1590/S0102-311X2009000700014

Murofuse, N. T., Abranches, S. S., \& Napoleão, A. A. (2005). Reflexões sobre estresse e Burnout e a relação com a enfermagem. Revista LatinoAmericana de Enfermagem, (13)2, 255-261. doi:10.1590/S010411692005000200019

Peixoto, L. (2016). Estresse afeta saúde gastrointestinal e causa sintomas como azia e diarreia. Minha vida. Recuperado de http://www.minhavida.com.br/saude/materias/17657-estresse-afetasaude-gastrointestinal-e-causa-sintomas-como-azia-e-diarreia

Pereira, J. G. \& Mello, F. (2017). Causas e efeitos do estresse no trabalho. Revista Interação, 146-164.

Pinto, F. M. \& Souza, P. C. Z. (2015). A atividade de trabalho de Motorista de Ambulância sob o ponto de vista ergológico. Revista Brasileira de Saúde Ocupacional, 25(131), 49-58. doi:10.1590/0303-7657000076113

Presidência da República. (1999). Decreto 3048/99 de 06 de maio de 1999. Regulamento do Ministério da Previdência Social. Recuperado de http://www.planalto.gov.br/ccivil_03/decreto/d3048.htm

Roazzi A., Carvalho A. D, Guimarães P. V. (2000). Análise da estrutura de similaridade da síndrome de burnout: Validação da escala "Maslach Burnout Inventory" em professores. Anais da VIII Conferência Internacional de Avaliação Psicológica - Formas e Contexto e $V$ Encontro Mineiro de Avaliação Psicológica: Teorização e Prática. Belo Horizonte.

Rozestraten, R. J. A. (1998). Psicologia do Trânsito, conceitos e processos básicos. São Paulo, SP: EPU/EDUSP.

Schaufeli, W. B. \& Enzmann, D. (1998). The burnout companion to study and practice: A critical analysis. London: CRC press.

Schaufeli, W. B. \& Janczur, B. (1994); Burnout among nurses: A Polish-Dutch comparison. Journal of Cross-Cultural Psychology, 95-113.

Tamayo, M. R. (1997). Relação entre a síndrome de burnout e os valores organizacionais no pessoal de enfermagem de dois hospitais públicos. (Dissertação de Mestrado). Universidade de Brasília, Brasília, Brasil.

Teixeira, J. A. C. (2004). Psicologia da Saúde. Análise Psicológica, 3(21), 441448.

Trigo, T. R., Teng, C. T., \& Hallak, J. E. C. (2007). Síndrome de burnout ou estafa profissional e os transtornos psiquiátricos. Revista Psiquiatria clínica, $34(5), 223-33$.

Varella, D. (2014). Doenças e sintomas: Síndrome de burnout. Recuperado de http://drauziovarella.com.br/letras/s/sindrome-de-burnout/ 
Volpato, C. D., Gomes, F. B., Castro, M. A., Borges, S. K., Jeisto, T., \& Benevides-Perera, A. M. T. (2003). Burnout em profissionais de Maringá. Revista Eletrônica de Interação Psy, 1(1), 102-111.

Sobre as autoras:

Caroline Estéfani Zanin é psicóloga e pós-graduada em Gestão Estratégia em Recursos Humanos pela Universidade do Oeste de Santa. Trabalha como Psicóloga Clínica e como tutora em cursos de Formação em Saúde Mental. zanin.caroline@hotmail.com.

Amanda Saraiva Angonese é psicóloga, pós-graduada e discente do Programa de Mestrado em Biociências e Saúde, da Universidade do Oeste de Santa Catarina. Trabalha como Professora no curso de Psicologia na Universidade do Oeste de Santa Catarina e como Psicóloga Clínica. amanda.angonese@unoesc.edu.br

C.E.Z. e A.S.A. participaram de forma equânime na realização da pesquisa e elaboração do artigo.

As autoras agradecem aos Motoristas do Transporte da Saúde pela disponibilidade e interesse em participar da pesquisa.

Recebido em: 23/06/2017 $1^{\text {a }}$ revisão em: 06/04/2018 Aceito em: 17/06/2018 